\title{
Consumo de estimulantes naturais por estudantes de medicina em uma instituição de ensino superior privada
}

\author{
Consumption of natural stimulants by medical students at a private educational institution \\ Consumo de estimulantes naturales por estudiantes de medicina en una institución \\ educativa privada
}

Maria Natália da Silva Montalvão ${ }^{1 *}$, Ana Celia Goes Melo Soares ${ }^{1}$, Rebeca dos Santos Sirqueira ${ }^{1}$, Rafael Ribeiro Almico Fraga ${ }^{1}$, Mayana Lula Andrade ${ }^{1}$, Antônio Souza Lima Junior'1.

\section{RESUMO}

Objetivo: Avaliar o uso de estimulantes naturais do sistema nervoso central pelos estudantes de graduação de Medicina, verificando o perfil dos usuários, a prevalência, a frequência, os motivos do uso e consequências. Métodos: Trata-se de um recorte de estudo descritivo, de abordagem quantitativa com 281 estudantes do curso em medicina do $1^{\circ}$ ao $6^{\circ}$ ano em Aracaju, na Universidade Tiradentes. Para a coleta de dados, utilizou-se um questionário com questões relacionadas aos estimulantes naturais. Os dados obtidos foram analisados por estatística descritiva com auxílio do software $\mathrm{R}$ Core Team 2020.0 estudo foi aprovado por Comitê de Ética em Pesquisa. Resultados: Evidenciou-se que: 93,6\% da amostra relatou já ter feito uso, em que $62,98 \%$ eram mulheres e $30,60 \%$ eram homens. Foi observado que $37,4 \%$ utilizam diariamente, $22,8 \%$ tinham forte desejo em utilizar e que o maior motivo para o uso está associado a tentativa de obter melhor desempenho acadêmico (63,9\%). Conclusão: Conclui-se que o consumo destas substâncias é prevalente e frequente no grupo estudado e que há forte desejo em consumi-las. A motivação para o uso foi tentar melhorar o desempenho acadêmico, no entanto, foram observados prejuízos em relação à qualidade do sono e ao humor.

Palavras-chave: Estimulantes do sistema nervoso central, Cafeína, Bebidas energéticas, Xantinas.

\begin{abstract}
Objective: To evaluate the use of natural stimulants of the central nervous system by undergraduate medical students in a private University of Sergipe (SE), checking the profile of users, prevalence, frequency, reasons for use and consequences. Methods: This is a descriptive study, with a qualitative and quantitative approach, with 281 medical students from the 1 st to the 6th year. For data collection, a questionnaire related to natural stimulants was used. The data obtained were analyzed using descriptive statistics with the aid of the R Core Team 2020 software. The study was approved by the Research Ethics Committee. Results: It was evidenced that: $93.6 \%$ of the sample reported had already used it, in which $62.98 \%$ were women and $30.60 \%$ were men. It was observed that $37.4 \%$ use it daily, $22.8 \%$ had a strong desire and that the biggest reason for use is associated with the attempt to obtain better academic performance (63.9\%). Conclusion: The conclusion is that the consumption of these substances is prevalent and frequent in the studied group and there is a strong desire to consume them. The motivation for use was to try to improve academic performance, however, losses were observed in relation to sleep quality and mood.
\end{abstract}

Keywords: Central nervous system stimulants, Caffeine, Energy drinks, Xanthines.

\section{RESUMEN}

Objetivo: Evaluar el uso de estimulantes naturales del sistema nervioso central por estudiantes universitarios de medicina de la Universidad privada de Sergipe (SE), verificando el perfil de usuarios, prevalencia, frecuencia, motivos de uso y consecuencias. Métodos: Este es un estudio descriptivo, con un enfoque cualitativo y cuantitativo, con 281 estudiantes de medicina del primer al sexto año. Para la recolección de datos, se utilizó un cuestionario con preguntas relacionadas con estimulantes naturales. Los datos obtenidos se analizaron utilizando estadísticas con el software R Core Team 2020. El estudio fue aprobado por el Comité de Ética en Investigación. Resultados: Se evidenció que: 93.6\% de la muestra informó haberlo usado, en el cual $62.98 \%$ eran mujeres y $30.60 \%$ hombres. Se observó que $37.4 \%$ lo usa diariamente, $22.8 \%$ tenía un fuerte deseo y que la razón más importante para el uso está asociada con intento de obtener un mejor rendimiento académico (63.9\%). Conclusión: Se concluye que el consumo de estas sustancias es frecuente en el grupo estudiado y que existe un fuerte deseo de consumirlas. La motivación para usar fue tratar de mejorar el rendimiento académico, sin embargo, se observaron pérdidas en relación con la calidad del sueño y estado de ánimo.

Palabras clave: Estimulantes del sistema nervioso central, Cafeína, Bebidas energéticas, Xantinas.

${ }^{1}$ Universidade Tiradentes (UNIT), Aracaju - SE. *E-mail: nataliamontalvao@hotmail.com 


\section{INTRODUÇÃO}

O ingresso dos jovens no ensino superior é um momento de muita felicidade, mas devido a mudanças no ambiente e nos costumes, pode tornar-se uma fase difícil (PEUKER AC, et al., 2006). A exposição a determinados fatores de risco pode desencadear o uso de substâncias, como a carga horária extensa, a necessidade de estudar frequentemente, a cobrança imposta pela sociedade e pelos próprios estudantes, a preocupação com o desempenho acadêmico, dentre outros (LEMOS KM, 2007).

O estilo de vida adotado pelos estudantes do ensino superior, especialmente estudantes de medicina, leva à necessidade de permanecerem ativos e acordados por um longo período de tempo para conseguir suprir a demanda de estudo. Além disso, pode causar prejuízos à saúde, como o sono irregular, pelo fato do mesmo ser uma função biológica fundamental na consolidação da memória, na recuperação do metabolismo energético cerebral e de energia.

Devido a essas importantes funções, os distúrbios do sono não apenas causam alterações cognitivas, como também nas funções sociais e físicas, comprometendo a qualidade de vida. Além disso, eles podem causar um declínio no desempenho acadêmico e produzir condições mais graves, como distúrbios mentais (BITTENCOURT LRA, et al., 2005; MÜLLERMR e GUIMARÃES, 2007; CARDOSO H, et al., 2009). É neste momento que encontramos o uso inadequado de drogas estimulantes pelos estudantes.

Outra questão relacionada é que o uso destas substâncias pode levar à dependência química, além de prejudicar o sono. Isso pode causar impacto negativo sobre os alunos ao afetar o raciocínio, o humor, o comportamento, além de reduzir a percepção e aumentar o estresse (SILVA LVER, et al., 2006; TOCKUS D e GONÇALVES PS, 2008). Os acadêmicos de Medicina, por exemplo, são um grupo suscetível ao abuso dessas substâncias com o objetivo de potencializar suas atividades intelectuais (NEWBURY-BIRCH D, 2000). Segundo Silveira VI, et al. (2015) o consumo de substâncias psicoestimulantes é considerado nocivo para a saúde devido aos efeitos produzidos no organismo. Estes mesmos autores revelaram que o consumo ocasional também pode levar à dependência.

Vale ressaltar que os estimulantes comumente consumidos podem ser classificados de duas maneiras diferentes: naturais e sintéticos. As xantinas ou estimulantes naturais, como a cafeína e a guaranina, são extraídos dos vegetais. De forma distinta, os estimulantes sintéticos, como a taurina e o metilfenidato são sintetizados em laboratório (ROMACH MK, et al., 2014). Dentre os naturais, a cafeína (trimetilxantina) é uma droga psicotrópica que estimula o sistema nervoso central, pertencendo ao conjunto de substâncias psicoativas que podem propiciar o abuso (GUERRA RO, et al., 2000). Geralmente, seus efeitos no corpo incluem a redução da fadiga e a melhora do estado de alerta, além de possibilitar o aumento da capacidade de executar determinadas tarefas.

Entre os estimulantes naturais, a cafeína é o mais usado pelos acadêmicos. Entretanto, altas doses dessa substância podem causar dependência e tolerância (KUDLOW PA, et al., 2013; SILVEIRA VI, et al., 2015). Em doses maiores, produz ansiedade, excitação e insônia e em consumidores habituais se desenvolve tolerância com necessidade de aumentar o consumo para obter os efeitos iniciais, além de dependência (REIS $E F, 2013)$. É consumida principalmente através de café, chá, chocolate, guaraná, mate e bebidas refrescantes, como cola e bebidas energéticas (ANDRADE FM, 2004).

Além disso, os energéticos contêm em sua composição elementos estimulantes e o consumo tem aumentado desde que surgiram no mercado (FINNEGAN D, 2003). Em princípio, foram desenvolvidos para aumentar a resistência física, a velocidade, a concentração, a sensação de bem-estar, o metabolismo, entretanto diminuem o sono (ALFONSO JP, et al., 2009; MENDOZA LA e PINEDA DUARTE AG, 2013). Seus principais componentes são água, carboidratos e cafeína. Vale ressaltar que a cafeína tem a função de estimular o sistema nervoso central e o carboidrato de fornecer energia (BONCE L, 2002). No entanto, a ingestão em altas doses ainda pode causar efeitos adversos, como taquicardia e ansiedade (BALLUS CA, et al., 2012).

Apesar do uso em larga escala de estimulantes naturais do sistema nervoso central, como cafeína e bebidas energéticas, ainda não está claro o perfil de uso por estudantes universitários, mais especificamente, 
do curso de medicina. Sendo assim, o objetivo do presente estudo foi investigar o perfil dos usuários, a prevalência, a frequência e os motivos do uso destas substâncias pelos estudantes de graduação em Medicina de uma Universidade particular de Sergipe (SE), além de avaliar suas possíveis consequências no sono e no humor.

\section{MÉTODOS}

O presente projeto foi aprovado pelo Comitê de Ética em Pesquisa envolvendo Seres Humanos de uma Universidade particular de Sergipe (SE), CAAE 12088919.4.0000.5371. Este é um recorte de um ensaio clínico sobre drogas lícitas, de caráter descritivo, transversal, abordagem quantitativa e de distribuição aleatória. Foram convidados para participar da pesquisa os estudantes universitários de ambos os sexos em uma instituição privada (Universidade Tiradentes), na cidade de Aracaju, estado de Sergipe.

Foram considerados como critérios de inclusão para esta amostra: estudantes maiores de 18 anos, cursando o período letivo de medicina em condição regular, que se dispuseram a responder o questionário e assinaram o termo de consentimento livre e esclarecido para participar da pesquisa. Ao ser considerado eticamente correto, iniciou-se o trabalho de campo, os estudantes foram informados do caráter anônimo dos dados e de que sua finalidade era exclusivamente científica. Eles também foram informados de que não eram obrigados a participar e foram excluídos os estudantes que não apresentavam os critérios descritos anteriormente.

Como a amostra do presente estudo foi estabelecida para uma população finita de 812 alunos no total de matriculados dos doze semestres de graduação de medicina, o cálculo da amostra populacional desejada foi de 254 alunos, que foi realizado com a formula de Gil (GIL AC, et al., 2008). Foram analisadas 281 respostas ao questionário, com um erro amostral de $5 \%$ e uma confiança de $95 \%$.

Foi mencionado que esta pesquisa teria como objetivo conhecer o modo de consumo de drogas lícitas, sendo assim os voluntários da pesquisa responderam perguntas referentes a frequência de uso, desejo do uso, possíveis prejuízos em suas atividades diárias e a tentativas de parar de utilizar, além de avaliar qualidade do sono e possíveis alterações do humor.

Para a análise, os dados foram organizados em uma planilha do programa Excel e depois processados no programa estatístico R Core Team 2020. Para a análise descritiva dos dados, foram utilizadas frequências, porcentagens, medias e desvio padrão. Para a analítica, especificamente em relação ao déficit cognitivo e demais variáveis categóricas, utilizou-se o teste Qui-Quadrado de Pearson.

As variáveis categóricas foram descritas por meio de frequência absoluta e relativa percentual. A aderência das variáveis contínuas à distribuição normal foi testada por meio do teste de Shapiro-Wilk. As diferenças nas medidas de tendência central foram testadas por meio do teste $T$ para amostras independentes (normal). $O$ nível e significância adotado foi de 5\%. Para efeito deste recorte os autores investigaram o perfil do uso de estimulantes naturais em estudantes de medicina desta amostra.

\section{RESULTADOS}

Foram aplicados 281 questionários em alunos de medicina de uma universidade particular do estado de Sergipe, distribuídos entre o primeiro ao décimo segundo período do curso. Do total de alunos pesquisados, $93,6 \%$ relataram já terem feito uso de estimulantes naturais (cafeína, guaraná, chá-mate, energéticos), sendo $45,4 \%$ do $1^{\circ}$ ao $6^{\circ}$ período. Do universo de mulheres entrevistadas, $62,98 \%$ faziam uso de estimulantes naturais, enquanto que no universo masculino $30,60 \%$ eram consumidores, ou seja, mais mulheres utilizam em relação aos homens.

Foi possível verificar o momento em que os acadêmicos utilizaram de forma mais frequente os estimulantes naturais. O maior valor identificado foi nos primeiros 2 anos de faculdade, com $31,2 \%$ havendo decréscimo após os 2 primeiros anos (19,8\%), havendo uma queda maior da frequência na época do internato $(8,7 \%)$, na qual o aluno possui uma vivência mais prática e mais próxima do dia a dia da profissão. O segundo foi o uso anterior à faculdade, com 29,7\%, que pode estar relacionado com a época do vestibular (Tabela 1). 
Tabela 1 - Momento que acadêmicos de medicina fizeram uso de estimulantes naturais de forma mais frequente.

\begin{tabular}{cc}
\hline Momento & $\mathbf{( \% )}$ \\
\hline Anterior a faculdade & 29,7 \\
Nos primeiro 2 anos de faculdade & 31,2 \\
Após os primeiros 2 anos de faculdade & 19,8 \\
No internato & 8,7 \\
Não aumentei a frequência/ Nunca fiz uso & 23,6
\end{tabular}

Fonte: Montalvão MN, et al., 2020.

Em relação à frequência de uso nos últimos 3 meses, notou-se que $37,4 \%$ usavam diariamente ou quase todos os dias, $15,3 \%$ semanalmente e $7,1 \%$ mensalmente. A partir destes resultados, é perceptível que o uso de estimulantes naturais é frequente nesta população. Já na segunda coluna da mesma tabela, foi questionado se havia um forte desejo ou urgência em consumir tal substância nos últimos 3 meses. Demonstrou-se que 22,8\% tinham este desejo diariamente ou quase todos os dias (Tabela 2).

Tabela 2 - Frequência de uso de estimulantes e de forte desejo de consumo do mesmo, nos últimos 3 meses.

\begin{tabular}{ccc}
\hline Frequência & Uso(\%) & Forte desejo(\%) \\
\hline Diariamente ou quase todos os dias & 37,4 & 22,8 \\
Semanalmente & 15,3 & 6,8 \\
Mensalmente & 7,1 & 3,6 \\
1 ou 2 vezes & 24,2 & 12,1 \\
Nenhum & 16 & 54,8 \\
\hline
\end{tabular}

Fonte: Montalvão MN, et al., 2020.

Sobre os motivos de uso, notou-se que o maior valor corresponde a necessidade de melhor desempenho acadêmico, com 63,9\%, 9,5\% para diversão e 8,4\% para redução de estresse (Tabela 3).

Tabela 3 - Motivos para o uso do estimulante natural.

\begin{tabular}{cc}
\hline Motivos & $(\%)$ \\
\hline Diversão & 9,5 \\
Redução de estresse & 8,4 \\
Socialização & 3,8 \\
Necessidade de melhora do desempenho acadêmico & 63,9 \\
Cefaleia & 6,5 \\
Outras dores & 1,1 \\
Nenhuma das opções anteriores & $27 \%$ \\
\hline
\end{tabular}

Fonte: Montalvão MN, et al., 2020.

Ao analisar as alterações do sono causadas por estimulantes naturais, os maiores valores corresponderam a dificuldade de iniciar o sono por pelo menos 20 minutos (39,2\%) e por sentir sonolência excessiva durante o dia $(31,6 \%)$. Outras alterações causadas foram "despertares noturnos com dificuldade de retornar ao sono" com $17,9 \%$ e "pesadelos e terror noturno" com 9,9\% (Tabela 4).

Tabela 4 - Alterações no sono causada pelo uso de estimulantes naturais.

\begin{tabular}{cc}
\hline Alteração & $(\%)$ \\
\hline Dificuldade de iniciar o sono por pelo menos 20 minutos & 39,2 \\
Sonolência excessiva durante o dia & 31,6 \\
Despertares noturnos com dificuldade de retornar ao sono & 17,9 \\
Pesadelos e Terror noturno & $9,9 \%$ \\
\hline
\end{tabular}

Fonte: Montalvão MN, et al., 2020. 
Os principais sintomas apresentados pelos acadêmicos após o uso de estimulantes naturais estavam associados a "medo, ansiedade e preocupação excessiva" com $30 \%$ e "deprimido ou desinteressado nas atividades na maior parte do dia" com $26,2 \%$. Outros sintomas relatados foram "humor persistentemente elevado, expansivo ou irritável" em $25,9 \%$, "irritado, zangado ou explosões de raiva frequentes" em $21,7 \%$, "comportamentos repetitivos ou atos mentais para prevenir ansiedade" em 19\% e "pensamentos ou imagens recorrentes indesejáveis" em 15,6\% (Tabela 5).

Tabela 5 - Sintomas apresentados após o uso de estimulantes naturais.

\begin{tabular}{cc}
\hline Sintomas & $(\%)$ \\
\hline Humor persistentemente elevado, expansivo ou irritável & 25,9 \\
Irritado, zangado ou explosões de raiva frequentes & 21,7 \\
Deprimido ou desinteressado nas atividades na maior parte do dia & 26,2 \\
Pensamentos ou imagens recorrentes indesejáveis & 15,6 \\
Comportamentos repetitivos ou atos mentais para prevenir ansiedade & 19 \\
Medo, ansiedade, preocupação excessivos & 30 \\
\hline
\end{tabular}

Fonte: Montalvão MN, et al., 2020.

\section{DISCUSSÃO}

Foi observado no nosso estudo, com uma média de idade de 22,8 anos, que os usuários de estimulantes naturais são $62,98 \%$ do sexo feminino e $30,60 \%$ do masculino. De maneira semelhante, o estudo de Sebastián EGR e Alfonso RCD (2014), também de estudantes universitários, traz que no estudo que abrangeu uma população mista de 293 estudantes, com idade média de 20,9 anos, sendo a prevalência de consumo de psicoestimulantes de $62,4 \%$ (183 alunos), com maiores resultados no sexo feminino $54,6 \%$ (160 alunas) em relação ao masculino com 45,3\% (133 alunos).

O estudo de Silveira VI, et al. (2015) também mostrou que o uso é maior em mulheres, sendo $33,62 \%$ contra $23,27 \%$ homens, no universo de $57 \%$ dos entrevistados. Da mesma forma, no estudo realizado por Penafort AG (2008), foi visto que 72,3\% (360 estudantes universitários) fazem uso regularmente, sendo a maioria do sexo feminino, com prevalência de 69,3\% (277 mulheres).

Ao contabilizar do $1^{\circ}$ ao 6 o período no nosso estudo, foi notável que $45,4 \%$ relataram já ter feito uso de estimulantes naturais (cafeína, guaraná, chá-mate, energéticos). Silveira VI, et al. (2015) traz prevalências semelhantes em estudo brasileiro realizado em Minas Gerais entre acadêmicos do $1^{\circ}$ ao $6^{\circ}$ período do curso de medicina esta prevalência foi de $57 \%$. Outra comparação estabelecida foi feita acerca da prevalência de uso dos alunos do quarto e quinto ano (corresponde ao $7^{\circ}, 8^{\circ}, 9^{\circ}, 10^{\circ}$ períodos), foi de $38 \%$ na nossa análise.

Um estudo publicado por Buchanan JC e Pillon SC (2008), entre estudantes de medicina do quarto e quinto ano em Honduras, mostrou uma prevalência de consumo de estimulantes naturais de $60,4 \%$. Os mesmos têm uma alta percentagem de repetência, sendo que Patologia a matéria de maior reprovação (30\%), seguido de Fisiologia, Psicologia e Farmacologia, com 10\% de reprovação. Por esse motivo, a sobrecarga nestes períodos em Honduras pode ser maior em relação ao 4ํ e $5^{\circ}$ ano do nosso estudo, favorecendo, dessa forma, um consumo maior de estimulantes naturais pela amostra de Honduras.

Em relação à frequência de uso, de acordo com o estudo de Silveira VI, et al. (2015) observa-se decréscimo na continuidade do uso por acadêmicos ao longo do ciclo básico. Dentre a amostra representativa, o primeiro ano apresentava um segmento majoritário no uso, $65,78 \%$ de usuários. Em seguida nota-se um decréscimo do uso no segundo ano, mas ainda representando a maior parcela, 64,10\%.

Constatou-se no terceiro ano uma perda da predominância no uso de psicoestimulantes, ao reportar apenas $41,02 \%$ da amostra do terceiro ano como utilizadora de estimulantes. Tais resultados corroboram com nossa análise, já que o maior valor identificado foi nos primeiros 2 anos de faculdade com $31,2 \%$, havendo decréscimo após os 2 primeiros anos $(19,8 \%)$, com uma queda maior da frequência na época do internato $(8,7 \%)$, na qual o aluno possui uma vivência mais prática e mais próxima do dia a dia da profissão. Estes dados podem revelar que 0 acadêmico se adaptou à necessidade da grade e ao padrão de ensino exigido. 
O maior motivo para o uso observado no presente estudo foi com a intenção de melhorar o desempenho acadêmico, correspondendo a $63,9 \%$ da amostra, e os motivos subsequentes foram $9,5 \%$ para diversão e $8,4 \%$ para redução de estresse. De acordo com o estudo de Mendes SV, et al. (2015), os principais motivos pelo uso de drogas estimulantes também eram aumentar a energia para melhorar o rendimento e o segundo, divergente em comparação à nossa análise, foi emagrecimento.

Vale ressaltar que houve esta distinção, pelo fato do estudo de Mendes SV, et al. (2015) envolver além dos estimulantes naturais, os estimulantes sintéticos e anorexígenos, os quais justificariam o emagrecimento. De maneira semelhante, o estudo de Sebastián EGR e Alfonso RCD (2014) também de estudantes universitários, verificou-se que a prevalência geral de consumo de psicoestimulantes para aumentar 0 desempenho acadêmico é de $62,4 \%$.

É possível perceber que em todos os estudos supracitados a alta frequência do uso por este motivo convergiu e estaria justificada pelas experiências do autor Ramos WP (2010), que demonstram que ingerir $300 \mathrm{mg}$ de cafeína levam a um aumento do rendimento intelectual e físico (RAMOS WP, 2010). Em relação ao consumo do café, o estudo de Alcântara JS (2018) demonstrou que os maiores motivos foram "o sabor", com $65,8 \%$ ( 85 alunos); "o hábito", com 63,5\% (85 alunos) e "intuito de manter-se acordado" em 58,9\% (76 alunos).

No entanto, é de suma importância a investigação da frequência e as consequências de uso de psicoestimulantes, pois esses provocam diversos efeitos colaterais indesejáveis e prejudiciais à saúde e à qualidade de vida dos estudantes, sendo a frequência de uso um fator que pode facilitar a relação de dependência (MENDES SV, et al., 2015).

No nosso estudo, ao realizar esta análise, notou-se que $37,4 \%$ usam diariamente ou quase todos os dias, $15,3 \%$ usam semanalmente, $7,1 \%$ usam mensalmente. Foi questionado se havia um forte desejo ou urgência em consumir tal substância nos últimos 3 meses e demonstrou-se que 22,8\% tinham este desejo diariamente ou quase todos os dias, confirmando a relação de tolerância e dependência nestes acadêmicos de medicina. No nosso estudo, foi possível observar os efeitos após o uso de estimulantes naturais em acadêmicos de medicina e os maiores valores estão associados a "medo, ansiedade e preocupação excessiva" com $30 \%$ e "deprimido ou desinteressado nas atividades na maior parte do dia" com $26,2 \%$.

Outros sintomas relatados foram "humor persistentemente elevado, expansivo ou irritável" em 25,9\%, "irritado, zangado ou explosões de raiva frequentes" em 21,7\%, "comportamentos repetitivos ou atos mentais para prevenir ansiedade " em 19\% e "pensamentos ou imagens recorrentes indesejáveis" em 15,6\%. No estudo de Alcântara JS (2018), 51\% (59 alunos) já sentiu algum mal estar, e 26\% (30 alunos) já pensou em parar de consumir. Dos motivos que os fizeram pensar na possibilidade de parar de consumir café, destacase a preocupação com a coloração dos dentes (38\%), gastrite e outros problemas gastrointestinais (37\%) e ansiedade (25\%).

Por outra análise, os autores Wang HR, et al. (2015) trazem que os consumidores habituais de café (cinco ou mais xícaras por dia) relatam que a abstenção Ihes causa irritabilidade, inquietação, nervosismo, cefaleia e dificuldade de rendimento no trabalho. Ao utilizar uma dose semelhante em não consumidores habituais de café ocorreu irritabilidade, nervosismo e dispepsia. Desta forma, pôde-se interpretar que o consumo habitual de grandes doses de cafeína produz tolerância e dependência, além de alterar negativamente o humor.

Além disso, Mendes SV, et al. (2015) também traz que os estimulantes podem alterar a função executiva e cognitiva dos seus usuários, pois os índices indicam uma piora na indução e qualidade do sono, assim como aumento da sudorese, o que gera desconforto na rotina do usuário. No estudo de Alcântara JS (2018), 33\% (43 estudantes) afirmaram ter problemas para dormir, sendo que desses estudantes $40 \%$ (17 alunos) acreditavam que esses problemas eram causados pela cafeína.

Corroborando com esta associação, Altermann AM, et al. (2008) traz que a cafeína provoca vários efeitos colaterais que podem limitar o seu uso ou trazer prejuízos à rotina executiva do paciente como insônia, dores de cabeça, irritação, ansiedade, prejuízo na memória e em alguns casos, até sangramentos gastrointestinais. Alguns desses prejuízos são relatados pelos participantes na pesquisa, como por exemplo, a qualidade do 
sono que não era muito boa em nenhum dos usuários. No nosso estudo, em relação ao sono, foi possível visualizar as alterações do sono causadas por estimulantes naturais, em que os maiores valores correspondem a dificuldade de iniciar o sono por pelo menos 20 minutos $(39,2 \%)$ e por sentir sonolência excessiva durante o dia (31,6\%).

Outras alterações causadas foram "despertares noturnos com dificuldade de retornar ao sono" com 17,9\% e "pesadelos e terror noturno" com 9,9\%. Corroborando com os resultados acima, o estudo de polissonografia realizado por Ho SC e Chung JW (2013) mostrou que os pacientes que consumiram café permaneceram mais tempo acordados (134 versus $104 \mathrm{~min}$ ) e tiveram maior número de despertares (5,4 versus 3,6), e, portanto, eficiência ao sono diminuída ( 70 versus $77 \%$ ) do que os que não consumiram. A densidade sono REM dos que ingeriram mais de $100 \mathrm{ml}$ de café foi significativamente menor do que os que tomaram menos do que 100 $\mathrm{ml}$. Diante disto, é possível perceber que estimulantes naturais influenciam significativamente o sono.

Deve ser incentivada a investigação sobre o uso de estimulantes naturais na população em geral e grupos populacionais em que o consumo se faça constante, como em estudantes universitários, a fim de traçar um perfil do uso discriminado e indiscriminado de estimulantes naturais no Brasil.

\section{CONCLUSÃO}

Concluiu-se que o consumo de estimulantes naturais tem sido prevalente entre os estudantes de medicina, sendo que a maior motivação esteve relacionada a tentativa de obter melhor desempenho acadêmico, havendo decréscimo ao longo dos períodos. Percebeu-se também o uso diário dos mesmos por parte de muitos estudantes, que podem apresentar forte desejo ou urgência em consumi-los. Como consequência observou-se queda da qualidade do sono, principalmente retardando o início e causando sonolência diurna excessiva, o que pode trazer prejuízos para a rotina, alterando as funções executivas e cognitivas. Além disso estas substâncias, se utilizadas em grande frequência, podem trazer sentimentos negativos como medo, ansiedade, depressão, desinteresse, o que, a longo prazo, pode levar a transtornos psiquiátricos.

\section{REFERÊNCIAS}

1. ALCÂNTARA JS. Avaliação do consumo diário de cafeína dos estudantes do Centro de Ciências da Saúde da Universidade Federal da Paraíba. Trabalho de Conclusão de Curso - Departamento de Nutrição. Universidade Federal da Paraíba, João Pessoa, 2018; 24p.

2. ALFONSO JP, et al. Factores de riesgo predictores del patrón de consumo de drogas durante la adolescencia. Anales de Psicología, 2009; 25(2): 330-338.

3. ALTERMANN AM, et al. A influência da cafeína como recurso ergogênico no exercício físico: sua ação e efeitos colaterais. Revista Brasileira de Nutrição Esportiva, 2008; 2(10).

4. ANDRADE FM. Avaliaçao de biomassa, clorofila, cafeína e tanino em llex paraguariensis Saint-Hilaire, crescendo sob sombreamento e pleno sol, 2004.

5. BALLUS CA, et al. Um método eficiente e rápido para determinação de cafeína em bebidas energéticas usando a técnica de eletroforese capilar (MEKC). Food Science and Technology, 2012; 32(2): 401-404.

6. BITTENCOURT LRA, et al. Sonolência excessiva. Revista Brasileira Psiquiatria, 2005; 27(1): 16-21.

7. BONCE L. Energy Drinks: Ayudan, Perjudican o Hiperenergizan. G-SE/Editorial Board/Dpto. Contenido. PubliCE, 2002.

8. BUCHANAN JC, PILLON SC. Uso de drogas entre estudiantes de medicina, Tegucigalpa, Honduras. Revista LatinoAmericana de Enfermagem, 2008; 16 (SPE): 595p.

9. CARDOSO HC, et al. Avaliação da qualidade do sono em estudantes de medicina. Rev. Bras. Educ. Med., 2009; 33(3), 349-355

10. DYRBYE LN, et al. Medical Students Distress: Causes, Consequences and Proposed Solutions. Mayo. Clin. Proc., 2005; 80(12): 1613-22.

11. FINNEGAN D. The health effects of stimulant drinks. Nutrition Bulletin, 2003; 28(2), 147p.

12. GIL AC, et al. Métodos e técnicas de pesquisa social. 6. ed. São Paulo: Editora Atlas SA, 2008; 55p.

13. GUERRA RO, et al. Cafeína e esporte. Revista Brasileira de Medicina do Esporte, 2000; 6(2): 60-62.

14. KUDLOW PA, et al. Cognitive Enhancement in Canadian Medical Students. Journal of Psychoactive Drugs, 2013; 45(4): 360-365.

15. HO SC, CHUNG JWY. (2013). The effects of caffeine abstinence on sleep: a pilot study. Applied Nursing Research, 2013; 26(2): 80-84.

16. LEMOS KM. Uso de substâncias psicoativas entre estudantes de medicina de Salvador. Revista Psiquiatria Clínica, 2007; 34(3):118-24.

17. MCCARTHY M, et al. Who is at risk and what do they know? Segmenting a population on their food safety knowledge. Food Quality and Preference, 2007; 18(2): 205-217. 
18. MENDES SV, et al. Estudo sobre o uso de drogas estimulantes entre estudantes de medicina. Revista Científica Multidisciplinar das Faculdades São José, 2015; (5)1.

19. MENDOZA LA, PINEDA DUARTE AG. Consumo de Psicoestimulantes para aumentar el rendimiento en el autoestudio, estudiantes de II-VI año carrera de medicina. Tese de Doutorado. Universidad Nacional Autónoma de Nicaragua, León, 2013; 63p.

20. MÜLLER MR, GUIMARÃES SS. Impacto dos transtornos do sono sobre o funcionamento diário e a qualidade de vida. Estudos de psicologia. Campinas, 2007; 24(4): 519-528.

21. NEWBURY-BIRCH D, et al. Factors influencing alcohol and illicit drug use amongst medical students. Drug and alcohol dependence, 2000; 59(2): 125-130.

22. PENAFORT AG. Padrão de consumo de café e de cafeína de um grupo populacional no nordeste brasileiro: risco à saúde ou não?, CE. Dissertação (Mestrado em Saúde Pública) - Centro de Ciências da Saúde. Universidade Estadual do Ceará, Fortaleza, 2008; 55p.

23. PEUKER AC, et al. Expectativas e beber problemático entre Universitários. Psicologia: Teoria e Pesquisa, 2006; 22(2):193-200.

24. RAMOS WPB. Abuso de drogas. São Paulo: Guanabara koogan, $2010 ;$ p. 207.

25. REIS EF. Plantas medicinais: um estudo da sua utilização popular no município de Rubim (MG). Ambiência, 2013; 9(3): 627-640.

26. ROMACH MK, et al. Human abuse liability evaluation of CNS stimulant drugs. Neuropharmacology, 2014; 87: 81-90.

27. SEBASTIÁN EGR, ALFONSO RCD. Prevalencia de consumo de sustancias psicoestimulantes y factores asociados, para aumentar el rendimiento académico, en estudiantes de primero a décimo nivel de la facultad de medicina de la pontificia universidad católica del ecuador desde noviembre de 2013 a enero de 2014. Dissertação (Título de cirurgião) - Pontificia Universidad Católica del Ecuador, Quito, 2014, 88-92p.

28. SILVA LVER, et al. Fatores associados ao consumo de álcool e drogas entre estudantes universitários. Revista Saúde Pública, 2006; 40(2): 280-288.

29. SILVEIRA VI, et al. Uso de psicoestimulantes por acadêmicos de medicina de uma universidade do Sul de Minas Gerais. Revista da Universidade Vale do Rio Verde, 2015; 13(2):186-192.

30. TOCKUS D, GONCALVES PS. Detecção do uso de drogas de abuso por estudantes de medicina de uma universidade privada. Jornada brasileira de psiquiatria, 2008; 57(3): 184-187.

31. WANG HR, et al. Caffeine-induced psychiatric manifestations: a review. International clinical psychopharmacology, $2015 ; 30(4): 179-182$. 